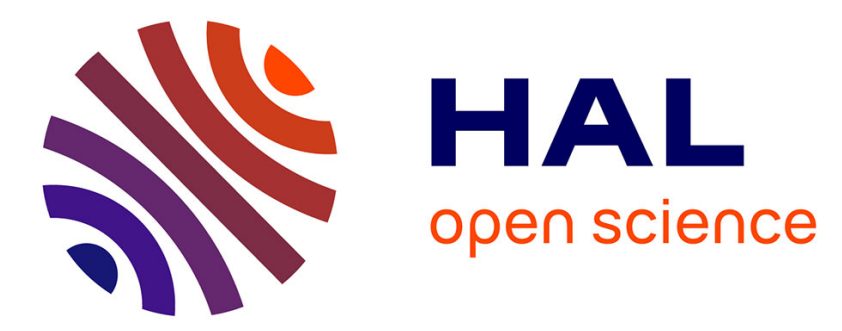

\title{
Multi-Character Physical and Behavioral Interactions Controller
}

\author{
Joris Vaillant, Karim Bouyarmane, Abderrahmane Kheddar
}

\section{To cite this version:}

Joris Vaillant, Karim Bouyarmane, Abderrahmane Kheddar. Multi-Character Physical and Behavioral Interactions Controller. IEEE Transactions on Visualization and Computer Graphics, 2017, 23 (6), pp.1650-1662. 10.1109/TVCG.2016.2542067 . hal-01246357

\section{HAL Id: hal-01246357 \\ https://hal.science/hal-01246357}

Submitted on 18 Dec 2015

HAL is a multi-disciplinary open access archive for the deposit and dissemination of scientific research documents, whether they are published or not. The documents may come from teaching and research institutions in France or abroad, or from public or private research centers.
L'archive ouverte pluridisciplinaire HAL, est destinée au dépôt et à la diffusion de documents scientifiques de niveau recherche, publiés ou non, émanant des établissements d'enseignement et de recherche français ou étrangers, des laboratoires publics ou privés. 


\title{
Multi-Character Physical and Behavioral Interactions Controller
}

\author{
Joris Vaillant, Karim Bouyarmane, and Abderrahmane Kheddar, Senior Member, IEEE
}

\begin{abstract}
We extend the quadratic program (QP)-based task-space character control approach — initially intended for individual character animation - to multiple characters interacting among each other or with mobile/articulated elements of the environment. The interactions between the characters can be either physical interactions, such as contacts that can be established or broken at will between them and for which the forces are subjected to Newton's third law, or behavioral interactions, such as collision avoidance and cooperation that naturally emerge to achieve collaborative tasks from high-level specifications. We take a systematic approach integrating all the equations of motions of the characters, objects, and articulated environment parts in a single QP formulation in order to embrace and solve the most general instance of the problem, where independent individual character controllers would fail to account for the inherent coupling of their respective motions through those physical and behavioral interactions. Various types of motions/behaviors are controlled with only the one single formulation that we propose, and some examples of the original motions the framework allows are presented in the accompanying video.
\end{abstract}

Index Terms-I.3 Computer Graphics, I.3.7 Three-Dimensional Graphics and Realism, I.3.7.a Animation; I.6 Simulation, Modeling, and Visualization, I.6.8 Types of Simulation, I.6.8.a Animation

\section{INTRODUCTION}

C HARACTER animation through physics simulation aims at generating interactive and physically plausible lowlevel character motions from high-level task objectives. Generally, the controller takes care of figuring out the necessary character's joint torques to realize desired tasks and feeds them to the simulator, that will in turn solve the forward dynamics, collision detection, and contact force problem with the given torques to produce the final motion in realtime.

Our approach builds on the well-studied QP-based method (see Section 2 for a brief review of previous studies). The character locomotion controller is formulated as follows:

$$
\begin{aligned}
& \underset{\substack{\text { accelerations } \\
\text { joint torques } \\
\text { contact forces }}}{ } \sum \text { quadratic objectives } \\
& \text { in }
\end{aligned}
$$$$
\text { subject to }\left\{\begin{array}{l}
\text { equation of motion } \\
\text { contact no-slip } \\
\text { friction cone limits } \\
\text { joint torque limits }
\end{array}\right. \text {. }
$$

The QP (1) is solved at every simulation time-step, the state of the character (positions and velocities) is updated after a given simulator applies the joint torques resulting from the optimization, and the QP (1) is executed for the

- The authors are with CNRS-University of Montpellier LIRMM Interactive Digital Humans group, 161 rue Ada, 34095 Montpellier, France. A. Kheddar is also with CNRS-AIST JRL UMI3218/RL, 1-1-1 Umezono, Tsukuba 305-8568, Japan

Manuscript received May 20, 2015; revised December 14, 2015 and XXXXX XX, 2016. next time-step in a new iteration. However, since there might exist multiple solutions to the given simulator's contact force problem, we shall prefer a more stable and replicable behavior, independent from the chosen external simulator, by integrating directly the accelerations resulting from the optimization. Doing so also allows us to use larger time-steps while preserving simulation stability. Nonetheless, bypassing the external simulator in this fashion does not hinder the targeted physics realism since the QP (1) acts itself as a physics simulator (simulating the dynamics equations of motion) provided that: (i) all the contacts are established, maintained, and released at controlled times, and (ii) unwanted collisions are avoided. Both (i) and (ii) are characteristics of our work.

In the previous works, the QP (1) was formulated exclusively for single character animation problems. Our contribution is to extend it to systems made of arbitrary numbers of interacting characters and objects, all in all ending up with the QP formulation (2). The rationale behind our idea, instead of simply using and composing independent individual QP character controllers, is to allow and ensure coherent interactions between the characters and objects in the scene. The motions of the characters are indeed coupled through the interactions between them. More specifically, we identified two main categories of interactions.

First the physical interactions that occur whenever characters are in physical contact with each other, resulting in the generation of contact forces in action/reaction pairs according to Newton's third law. In our extension of the QP, we propose an ordering scheme of the components of the systems and their respective forces so as to keep one and only one representative of each action/reaction pair for a minimal set of optimization variables.

The second category of interactions that implicitly create a coupling between the motions is what we called behav- 


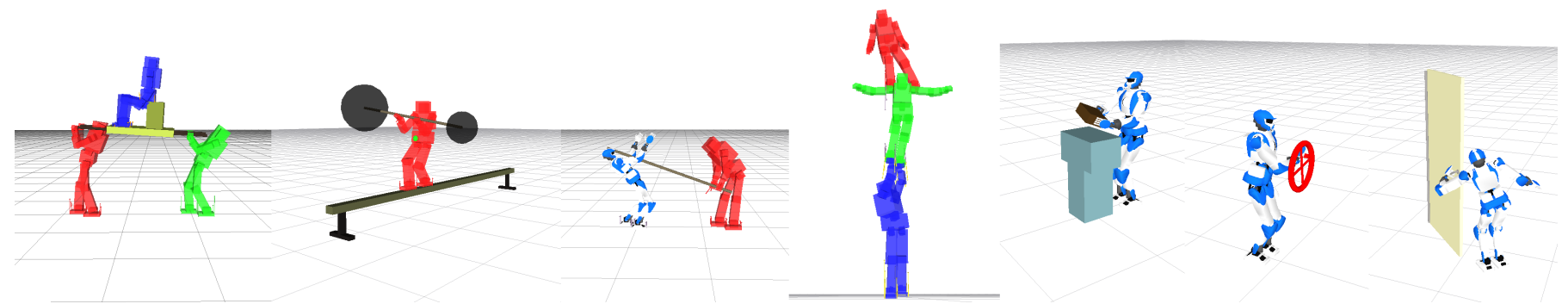

Fig. 1. A selection of example animation scenarios that can be modeled in our framework, screenshots from the accompanying video.

ioral interactions and are themselves of two kinds: collision avoidance constraints and collaborative tasks. Collisionavoidance constraints impose for the characters to be "aware" of the presence of each other in their close vicinity and to "predict" each other's motion in order to avoid collisions. The centralized QP ensures such awareness and interpredictability, since all the motions are computed together at once by one central controller. Collaboration is also readily encoded in the extension of the QP, by simply writing tasks that are automatically dependent on the motions of the characters involved in the cooperation action. The centralized $\mathrm{QP}$ will ensure that the actions are coordinated in an optimal synergy for performing the task.

Accounting for all these interactions that result in the coupling of the motions, we propose the following compact and easy-to-implement integrated multi-character $\mathrm{QP}$ controller:

$$
\begin{aligned}
& \underset{\text { min }}{\min } \sum \text { quadratic objectives } \\
& \text { multi-character joint torques } \\
& \text { minimal set of contact forces }
\end{aligned}
$$

$$
\text { subject to }\left\{\begin{array}{l}
\text { system of equations of motion } \\
\quad \text { coupled through Newton's third law } \\
\text { contact no-slip (with the fixed environment) } \\
\text { contact no-slip (multi-character interaction) } \\
\text { friction cone limits } \\
\text { joint torque limits } \\
\text { joint position and velocity limits } \\
\text { collision avoidance }
\end{array}\right.
$$

In the QP (2) our technical contributions are highlighted. The red-colored components are contributions pertaining to the formulation of the problem as a multi-character system. The blue-colored components are independent of the multi-character nature of the problem and can as well be incorporated into existing single character controllers. We experimentally confirm that the centralized brute-force approach consisting in solving one large integrated QP is computationally tractable, by having implemented and executed our framework on a standard laptop computer and generated our motions in real-time or close to real-time.

The presentation of the method is structured in Section 3 as follows: we formulate the multi-character problem (redcolored parts of (2)) with the system of equations of motions in Section 3.1 and the contact no-slip constraint in Section 3.2. Then, we detail the collision-avoidance constraint in Section 3.3 and the joint position and velocity limits among others in Section 3.4. In order to make the paper self-contained, we recall the rest of the components of the QP that we borrow as such from the literature without particular alteration in our method. Those are the friction cone and torque limits in Section 3.4 and the formulation of the quadratic objectives/tasks in Section 3.5. In these latter two sections we only reproduce existing works from the literature. The final form of problem (2) is finally formulated as Equation (35) in Section 3.6. The rest of the paper presents the results in the form of a description of the accompanying video in Section 4, and a discussion and conclusion in Section 5 .

\section{Literature Review and Related Work}

Early work on human character physics simulation achieved impressive results using action-specific controllers [1], [2], [3]. In these seminal works a controller is designed for a given human skill (e.g. running, diving, pole-vaulting, biking) and per-joint PD servos track the designed motion in physics simulation. This approach requires the skillful design of a new controller for every new action, and later works would apply the joint-space approach to broader or more parametrizable classes of actions [4], [5], [6]. Taskspace approaches have been proposed as an alternative, adapting work done in robotics [7], [8], [9], [10], see e.g. [11].

The task-space formulation is either based on strict hierarchy prioritization - using null-space projectors; or on weighted hierarchy - combining all the tasks in a single QP; or on a mix of both. Our work is mostly inspired from previously proposed QP-based motion controllers, in particular the two works [12], [13], and to a lesser extent [14]. [12] initially proposed a framework for achieving standing balance control of physically simulated characters in a given contact configuration with the environment, which allows to either target a static reference posture or to track motion capture data performed from a fixed stance. This work was followed by [15], [16] that extended it to periodic walking.

Based on a similar QP formulation, but with a hybrid priority-weighted policy for the objectives/constraints, [13] proposed a more general-purpose controller for the locomotion of various biped characters. Momentum objective as proposed in [17] and later used in [18] was included and shown to yield "natural-looking" behaviors for walking or jumping. The QP controller was in this work coupled 
with a finite-state machine (FSM) that builds the appropriate instance of the prioritized-QP problem to control a given phase of the locomotion. In our present work, we opted for the weighted approach and similarly used FSM decompositions of the various phases of our motions. That controller in [13] was also used as a low-level controller that realizes a higher-level plan in [19], showcasing robust bipedal walking and running simulations.

The authors in [14] proposed a slightly different formulation of the problem, which is directly in the joint position space. They demonstrate a wide variety of character balancing behaviors, with one of them involving a cooperation between two characters. The capabilities of our framework and those of [14] seem similar in that regard. It is however unclear and not detailed in the paper how the cooperation is effectively achieved and if it was specifically designed for the example motion. Instead, we focus on the systematic modeling of arbitrary systems in the most general setting, e.g. object manipulation, and on our novel behavioral interactions. Another advantage appears in comparison to the non real-time aspect of [14]. Yet [14] provides more advanced FSM design methodologies than ours since we mainly focus on the low-level controller. Our controller can for example be used with the FSMs of [14].

Trajectory optimization approaches, on the other hand, allow to synthesize broader ranges of parametrizable motions at the expense of little or no interactivity and high computational costs which often makes them unadapted to real-time applications, but still achieving a high degree of realism for original highly dynamic motions [20], [21], [22]. All these works however are mainly about the locomotion of one character in the world and do not integrate, with that locomotion behavior, a manipulation behavior component [11], [23], [24], [25], [26], a cooperative behavior component [27], [28], [29], [30], a quadruped walking behavior component [31], a dexterous hand component [32], to cite a few examples among a vast body of existing works and approaches in these fields.

Recently [33], [34] introduced contact-invariant optimization (CIO) motivated by our same expressed desire of proposing a framework capable of embracing a wide variety of classes of character motions at once [35]. They succeeded in demonstrating that their approach enables to yield (i) locomotion behaviors beyond periodic biped walking, e.g. climbing, crawling, standing-up motions, etc. (ii) various hand dextrous manipulations, and (iii) objectmanipulation and multiple character cooperating. Though this was done in an offline trajectory optimization approach which prevents real-time interactive control possibilities and with simplified physics, our present work is inspired by the same philosophy of generality in the targeted character motion instances. We had previously proposed such a unification approach of humanoid behaviors in non real-time motion generation contexts. Those are the static single posture generation problem for multiple robot systems in [36], formulated as an inverse kinematics problem integrating all the robots and objects at once, and the planning problem of a sequence of such static postures in [35]. We propose now a real-time controller based on the same philosophy.

To sum up this section, our work can be seen as reconciling different aspects of the works reviewed here in what

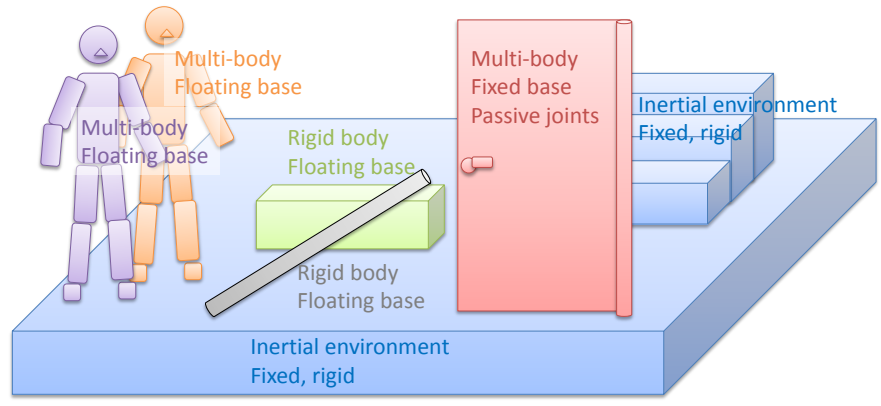

Fig. 2. Various instances of subsystem types that combine together to create the full system controlled as a whole in the animation scene. Each individual subsystem is represented in a different color.

thus constitutes a novel approach. Namely, we fusion the aspects of real-time interactive physics simulation proposed in [12], [13] with the general motion planning philosophy adopted in [35] [33], or, in other words, we target the same level of generality attained in the latter using the more flexible, interactive-control-enabling approach of [12], [13].

\section{Method}

\subsection{Equation of Motion}

Our method considers all the interacting characters, objects, and the environment in the scene as one system. Let us denote $n$ the number of all identified independent subsystems in the scene. One such independent subsystem can be a character, a rigid object (e.g. manipulated box), an articulated part of the environment (e.g. a door, a valve, etc.), see Fig. 2. We index them with the variable $i$ in $\{1, \ldots, n\}$, and we use the index $i=0$ for the rest of the rigid inertial environment (ground, walls, stairs, etc.). Every subsystem $i \in\{1, \ldots, n\}$ can be modeled as either a fixed-base or a floating-base articulated kinematic tree with configuration vector $q_{i} \in \mathbb{R}^{\mu_{i}}$ (which includes the free-floating base position/orientation if any and the joint angles if any), and behave following their respective $\mathrm{EOM}^{1}$

$$
M_{i}\left(q_{i}\right) \ddot{q}_{i}+N_{i}\left(q_{i}, \dot{q}_{i}\right)=J_{\mathrm{all}, i}\left(q_{i}\right)^{T} f_{\mathrm{all}, i}+S_{i} \tau_{i},
$$

where $\tau_{i} \in \mathbb{R}^{a_{i}}$ is the vector of torques acting on the actuated DOFs of the subsystem $\left(a_{i}=0\right.$ for a manipulated object and for passive articulated part of the environment) and $S_{i} \in \mathbf{M}\left(\mu_{i}, a_{i}\right)$ is the selection matrix that maps the dimension of $\tau_{i}$ to that of $q_{i}$ by extending $\tau_{i}$ with zeros at the indexes of the non-actuated DOFs (which include the free-floating base DOFs if any). The subsystem is supposed to be subjected to the action of a set of $\nu_{i}$ punctual contact forces $f_{\text {all }, i} \in \mathbb{R}^{3 \nu_{i}}$ with respective Jacobians at the corresponding contact points $J_{\text {all }, i} \in \mathbf{M}\left(3 \nu_{i}, \mu_{i}\right) . M_{i}$ and $N_{i}$ are respectively the mass matrix and the term regrouping the non-linear effects and the gravity. Equation (3) reduces to the Newton-Euler EOM for a rigid body subsystem (e.g. a manipulated object).

1. The notations of the paper are consistent with the conventional identification of vectors as column matrices (and not as row matrices) $\mathbb{R}^{r} \equiv \mathbf{M}(r, 1)$, meaning that $\left(\lambda_{1}, \ldots, \lambda_{r}\right) \equiv\left(\begin{array}{lll}\lambda_{1} & \ldots & \lambda_{r}\end{array}\right)^{T}$ and in particular that $\left(\lambda_{1}, \ldots, \lambda_{r}\right) \not \equiv\left(\begin{array}{lll}\lambda_{1} & \ldots & \lambda_{r}\end{array}\right) . \mathbf{M}(\alpha, \beta)$ denotes the set of real matrices of $\alpha$ rows and $\beta$ columns. 
Each contact force applied at subsystem $i$ is either applied by the inertial environment or by another subsystem $j$ and thus appears, in the latter case, with an opposite sign in subsystem $j$ 's EOM according to Newton's third law. We thus rewrite all Equations (3) in the following forms:

$$
\begin{aligned}
& M_{i}\left(q_{i}\right) \ddot{q}_{i}+N_{i}\left(q_{i}, \dot{q}_{i}\right)= \\
& \quad J_{0, i}\left(q_{i}\right)^{T} f_{0, i}+J_{1, i}\left(q_{i}\right)^{T} f_{1, i}-J_{2, i}\left(q_{i}\right)^{T} f_{2, i}+S_{i} \tau_{i},
\end{aligned}
$$

where $f_{0, i}$ are the contact forces applied by the environment on subsystem $i, f_{1, i}$ are the contact forces applied by subsystems $j \in\{1, \ldots, i-1\}$ on subsystem $i$, and $f_{2, i}$ are the forces applied by subsystem $i$ on subsystems $j \in\{i+1, \ldots, n\}$.

Let $F_{0}, F_{1}, F_{2}$ be respectively the stacked vectors of all the forces $f_{0, i}$ 's, $f_{1, i}$ 's, and $f_{2, i}$ 's, i.e.

$$
F_{k}=\left(f_{k, i}\right)_{1 \leq i \leq n}, k=0,1,2 .
$$

Since, $\forall i \in\{1, \ldots, n\}$, all the forces in $f_{2, i}$ appear at some position in some of the $f_{1, j}$ forces, with $j$ in a subset of $\{1, \ldots, n\}$, we can write $f_{2, i}=\phi_{i} F_{1}$ where $\phi_{i}$ is a selection matrix that selects the adequate elements in $F_{1}$ and reorders them into $f_{2, i}$.

Equations (4) thus take the following forms:

$$
\begin{aligned}
& M_{i}\left(q_{i}\right) \ddot{q}_{i}+N_{i}\left(q_{i}, \dot{q}_{i}\right)= \\
& J_{0, i}\left(q_{i}\right)^{T} f_{0, i}+J_{1, i}\left(q_{i}\right)^{T} f_{1, i}-J_{2, i}\left(q_{i}\right)^{T} \phi_{i} F_{1}+S_{i} \tau_{i} .
\end{aligned}
$$

The common variable $F_{1}$ binds together all the Equations (6). This binding transcripts the coupling of the motions through the physical interactions among the subsystems. By denoting $q=\left(q_{1}, \ldots, q_{n}\right)$ and $\tau=\left(\tau_{1}, \ldots, \tau_{n}\right)$ and by stacking together all the elements of Equations (6):

$$
\begin{aligned}
M(q) & =\operatorname{diag}\left(M_{1}\left(q_{1}\right), \ldots, M_{n}\left(q_{n}\right)\right) \\
J_{k}(q) & =\operatorname{diag}\left(J_{k, 1}\left(q_{1}\right), \ldots, J_{k, n}\left(q_{n}\right)\right)_{k=0,1,2} \\
S & =\operatorname{diag}\left(S_{1}, \ldots, S_{n}\right) \\
\Phi & =\left(\begin{array}{c}
\phi_{1} \\
\vdots \\
\phi_{n}
\end{array}\right) \\
N(q, \dot{q}) & =\left(\begin{array}{c}
N_{1}\left(q_{1}, \dot{q}_{1}\right) \\
\vdots \\
N_{n}\left(q_{n}, \dot{q}_{n}\right)
\end{array}\right)
\end{aligned}
$$

we can rewrite Equations (6) as one EOM of the full system that makes up our animation scene:

$$
M(q) \ddot{q}+N(q, \dot{q})=J_{0}(q)^{T} F_{0}+\left(J_{1}(q)^{T}-J_{2}(q)^{T} \Phi\right) F_{1}+S \tau
$$

Note: $\Phi$ defined in (10) is a square permutation matrix ${ }^{2}$, thus in particular an orthogonal matrix $\Phi^{T} \Phi=I$, since it maps the triple position of every internal contact force of the system in the stacked vector $F_{1}$ to its Newton's third law counterpart triple position that uniquely exists in the

2. More precisely, if $3 \kappa$ is the size of $F_{1}$, where $\kappa$ is the total number of punctual forces from our ordering convention stacked into $F_{1}$, then $\Phi$ is of the form $\Phi=P \otimes I_{3}$ where $P$ is a permutation matrix of size $\kappa \times \kappa$ and $I_{3}$ is the $3 \times 3$ identity matrix. The operator $\otimes$ denotes the Kronecker product.

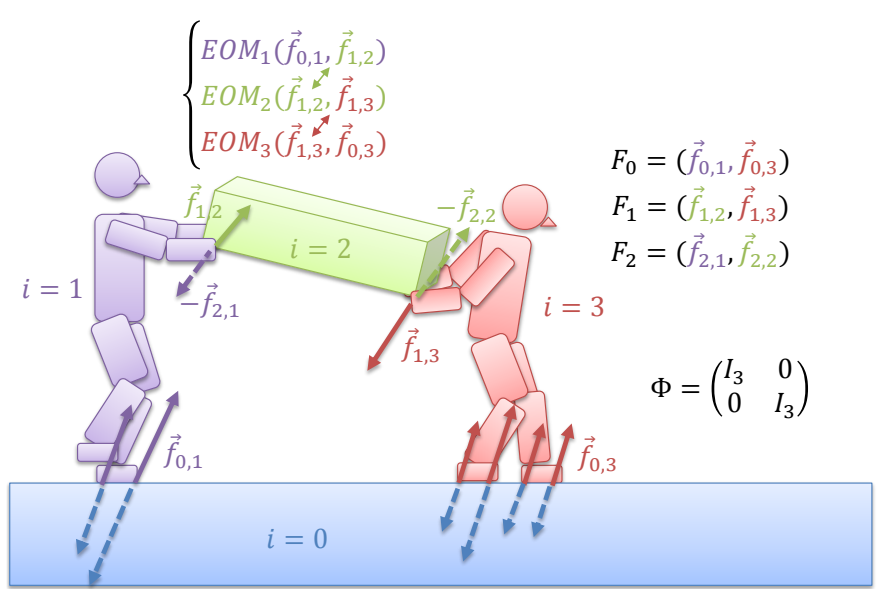

Fig. 3. Deriving the entire system's EOM from the individual subsystems respective EOMs. Each subsystem is shown in a different color and the contact forces applied on a subsystem are shown in the same color as the subsystem. Contact forces that are left as optimization variables from our ordering convention and that effectively appear in the QP problem are shown as solid arrows (they make up the variables $F_{0}$ and $F_{1}$ ), while dashed arrows represent the corresponding reaction forces. The relation $F_{2}=\Phi F_{1}$ encodes Newton's third law, and the forces $-F_{2}$, by convention, do not independently appear in the QP. The upper brace EOM shows how the three individual EOMs are coupled through the contact forces $f_{1,2}$ and $f_{1,3}$, i.e. through the QP variable $F_{1}$.

stacked vector $F_{2}$. The relation $F_{2}=\Phi F_{1}$ encodes Newton's third law in the whole system and in (12). $F_{2}$ does not appear in this equation anymore and thus $\left(F_{0}, F_{1}\right)$ is the minimal set of force optimization variables we keep in the formulation. See Fig. 3 for a simple case example.

\subsection{Contact No-Slip}

In addition to (12), the consistency of the physical interactions that occur in the scene is ensured by enforcing the following contact no-slip constraints:

$$
\begin{gathered}
J_{0}(q) \dot{q}=0, \\
J_{2}(q) \dot{q}=\Phi J_{1}(q) \dot{q},
\end{gathered}
$$

Equation (13) is usually written in existing single character QP controllers, encoding the zero-velocity condition of the contact points of the subsystems with the inertial environment. Equation (14) however is exclusive to the multi-character system and encodes the zero-relative-velocity condition of all pairs of contact points belonging to pairs of subsystems in contact. The mapping $\Phi$ introduced in the previous section appears to be helpful here and allows a very compact encoding of this condition. It expresses that the mapping of the contact forces $F_{2}=\Phi F_{1}$ is conserved for the contact point velocities obtained from the stacked Jacobian matrices through the principle of virtual work. Note that since $\Phi^{-1}=\Phi^{T}$, Equation (14) is equivalent to

$$
\left(J_{1}(q)^{T}-J_{2}(q)^{T} \Phi\right)^{T} \dot{q}=0
$$

and, consequently, $F_{1}$ appears to be the Lagrange multiplier associated with this constraint in (12), which can thus be interpreted as the Lagrange's equation of the whole system. 
Equations (13) and (14) are time-differentiated to obtain constraints on the accelerations compatible with the QP:

$$
\begin{gathered}
J_{0}(q) \ddot{q}+\dot{J}_{0}(q) \dot{q}=0 \\
\left(\Phi J_{1}(q)-J_{2}(q)\right) \ddot{q}+\left(\Phi \dot{J}_{1}(q)-\dot{J}_{2}(q)\right) \dot{q}=0 .
\end{gathered}
$$

These formulations are prone to numerical instability (also reported in [13]) so we replace them in our implementation with a more stable behavior-yielding formulation as follows. For every contact between subsystem $i$ and the fixed inertial environment, let us denote $v_{0, i}$ the $6 \mathrm{D}$ linear and angular velocity and $\mathcal{J}_{0, i} \in \mathbf{M}\left(6, \mu_{i}\right)$ the corresponding linear and angular Jacobian of the contact link of the subsystem; the no-slip constraint for this contact link is written as:

$$
\mathcal{J}_{0, i} \ddot{q}_{i}+\dot{\mathcal{J}}_{0, i} \dot{q}_{i}=-\frac{v_{0, i}}{\Delta t},
$$

where $\Delta t$ is the integration time-step. These constraints (18) replace the constraint (16).

For a contact between subsystems $i$ and $j$, let $v_{1, i}$ and ${ }^{1, i} v_{2, j}$ denote respectively the $6 \mathrm{D}$ velocity of the contact link of subsystem $i$ ("link 1") and the $6 \mathrm{D}$ velocity of the contact link of subsystem $j$ ("link 2") transformed in link 1 frame and expressed at the same reference point (in the sequel we denote it for brevity only as $v_{2, j}$ ). Finally let $\mathcal{J}_{1, i}$ and ${ }^{1, i} \mathcal{J}_{2, j}$ (for brevity again denoted $\mathcal{J}_{2, j}$ ) denote the corresponding $6 \mathrm{D}$ Jacobians. A more stable behaviour than that of (17) is obtained by writing:

$$
v_{1, i}+\left(\mathcal{J}_{1, i} \ddot{q}_{i}+\dot{\mathcal{J}}_{1, i} \dot{q}_{i}\right) \Delta t=v_{2, j}+\left(\mathcal{J}_{2, j} \ddot{q}_{j}+\dot{\mathcal{J}}_{2, j} \dot{q}_{j}\right) \Delta t
$$

Yet, even this latter formulation might lead to numerical inaccuracies since the transformation from the frame of link 2 to that of link 1 is not constant over time (due to small perturbations leading to loss of contact), so we propose a refined version of (19) that ultimately proves stable in our experience (for integration time-steps $\Delta t$ ranging between $5 \mathrm{~ms}$ and up to $33 \mathrm{~ms}$ )

$$
\begin{aligned}
v_{1, i}+\left(\mathcal{J}_{1, i} \ddot{q}_{i}+\dot{\mathcal{J}}_{1, i} \dot{q}_{i}\right) \Delta t & -v_{2, j}-\left(\mathcal{J}_{2, j} \ddot{q}_{j}+\dot{\mathcal{J}}_{2, j} \dot{q}_{j}\right) \Delta t \\
= & -\frac{\operatorname{Err}\left(2, j X_{1, i}^{\mathrm{ref}}{ }^{1, i} X_{2, j}\right)}{\Delta t},
\end{aligned}
$$

where $\operatorname{Err}\left({ }^{2, j} X_{1, i}^{\mathrm{ref}} 1, i X_{2, j}\right)$ expresses the $6 \mathrm{D}$ error between (a) ${ }^{1, i} X_{2, j}$, the current transformation of the link 2 frame in the link 1 one, and (b) ${ }^{1, i} X_{2, j}^{\mathrm{ref}}$, that same transformation in the reference ideal situation where the contact is perfectly established (initial state of the contact). ${ }^{3}$

\subsection{Collision Avoidance}

Collision avoidance is one of the behavioral interactions between the subsystems of the scene that create an implicit coupling of their motions. The collision-avoidance

3. If ${ }^{B} X_{A}$ denotes a $6 \mathrm{D}$ transformation matrix from frame $A$ to frame $B,{ }^{B} X_{A}=\left(\begin{array}{cc}R & 0 \\ -(R r) \times & R\end{array}\right)$, where $R$ and $r$ denote respectively the rotation matrix and translation vector from frame $A$ to frame $B$, $\operatorname{Err}\left({ }^{B} X_{A}\right)$ is defined as the $6 \mathrm{D}$ vector $\operatorname{Err}\left({ }^{B} X_{A}\right)=\left(\begin{array}{c}\ln R \\ r\end{array}\right)$, where $\ln R$ is defined as the the angular velocity vector that yields $R$ over a unit time, i.e $R=\exp (w t)$ with $t=1$. constraint is however not exclusive to the multi-character problem and can also be used in single character applications for avoiding static or moving obstacles.

To the best of our knowledge, no previous QP-based approach proposed in the literature dealt with this kind of constraint at such low level, and we believe that detailing it here would constitute an original addition to state-ofthe-art QP-based controllers. Existing collision-avoidance approaches are usually encoded at higher levels with predefined, known, obstacle trajectories or with predicted obstacle motions, e.g. [14], [30]. Our approach does not need any pre-computation or prediction of trajectories and acts in a reactive fashion to any currently occurring motions. Recent work, that also includes collision-avoidance constraints in a QP-based control, can be found in [37]. Other related work incorporates a reactive collision avoidance scheme similar to the one we use here in an inverse-kinematics-based motion reconstruction from motion capture data [38].

A collision-avoidance constraint in our framework can be written between any pairs of bodies in the scene, whatever subsystem they belong to (including the inertial environment). The distance computation method we use is an implementation of the Gilbert, Johnson and Keerthi (GJK) algorithm, as detailed in [39]. The GJK algorithm is based on so-called support functions that allow, at a given configuration of two convex bodies, to return two witness points belonging to the surfaces of each body for which the distance is equal to the distance between the two bodies. These witness points move along the surfaces of the two bodies as their configuration change over time. We apply the strictly-positive distance constraint on these two moving points, thus guaranteeing the satisfaction of the collisionavoidance constraint between the two considered convex bodies they belong to. Non-convex bodies are decomposed into convex components (or approximation thereof if no such decomposition exists) and the constraint is applied on the convex components.

The formulation of the collision-avoidance constraint relies on velocity damping initially proposed in robotics applications [40], [41]. Let us consider two bodies of the scene belonging respectively to subsystems $i$ and $j$ for which we would like to write the collision-avoidance constraint. The distance $d$ between the two bodies is

$$
d=\sigma\left\|p_{1, i}-p_{2, j}\right\|,
$$

where $p_{1, i}$ and $p_{2, j}$ are the two witness points, and $\sigma=+1$ is there is no collision and $\sigma=-1$ if $\delta$ is an inter-penetration distance. A basic velocity damper behaviour is obtained through the following inequality

$$
\dot{d} \geq-\xi \frac{d-\delta_{s}}{\delta_{i}-\delta_{s}}
$$

where $\xi, \delta_{s}$, and $\delta_{i}$ are fixed parameters representing respectively the damping factor, the security distance, and the influence distance below which the constraint is activated. A QP-compatible version can be written as

$$
\ddot{d} \geq \frac{1}{\Delta t}\left(-\xi \frac{d-\delta_{s}}{\delta_{i}-\delta_{s}}-\dot{d}\right) .
$$

Denoting $u=\left(p_{1, i}-p_{2, j}\right) / d$ the unit vector between $p_{1, i}$ and $p_{2, i}$, the derivative $\dot{d}$ is obtained as $\dot{d}=\left(\dot{p}_{1, i}-\right.$ 


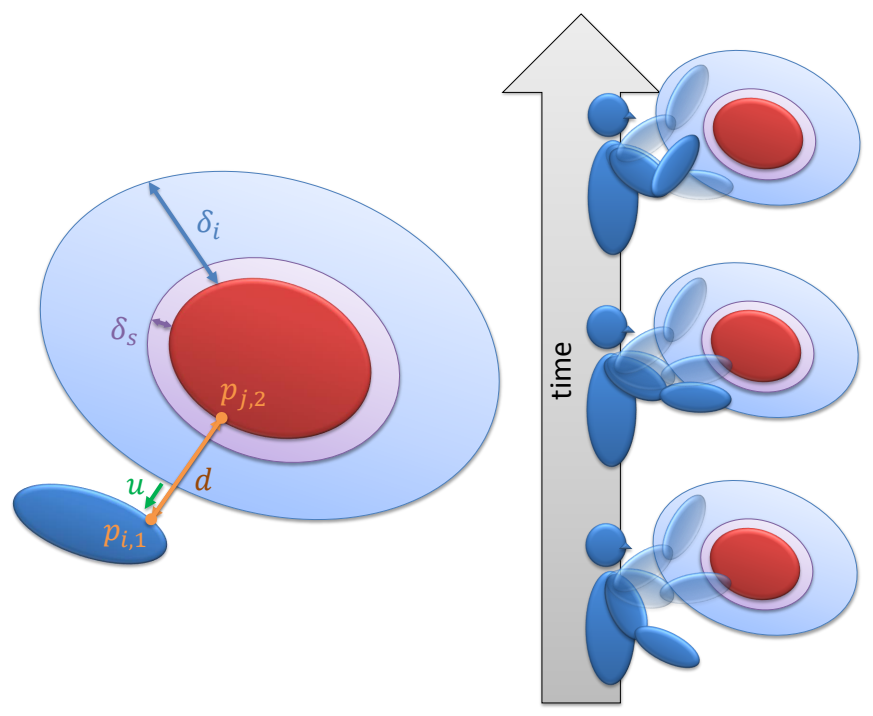

Fig. 4. Illustration of the collision-avoidance method. In the left figure, the blue and red bodies must avoid colliding. The thin purple layer wrapping the red body represents the security "forbidden" zone from which we consider that collision has occurred (this security distance can just be reduced to zero), the light blue zone shows the influence distance from entering which the constraint is activated and starts influencing the motions of the bodies. The right figure is decomposed in three sequential frames. The bottom frame shows the initial position and expected motion of the arm performing a reaching task. This expected motion will collide with the red obstacle. Between the bottom and the middle frame, the motion of the arm occurs outside of the influence zone, so the motion is not affected. In the middle frame, the arm enters the influence zone and the constraint is activated, leading to the deviation of the motion that is shown in the top frame, in which the arm avoids collision by avoiding the purple security layer around the red obstacle.

$\left.\dot{p}_{2, j}\right)^{T} u$. Finally denoting $J_{1, i}^{\mathrm{lin}}$ and $J_{2, j}^{\mathrm{lin}}$ respectively the linear (translational) Jacobians of subsystems $i$ and $j$ at $p_{1, i}$ and $p_{2, j}$, equation (23) takes the following final form that we add as a constraint to the QP

$$
\begin{aligned}
& u^{T}\left(J_{1, i}^{\operatorname{lin}} \ddot{q}_{i}+\dot{J}_{1, i}^{\operatorname{lin}} \dot{q}_{i}-J_{2, j}^{\operatorname{lin}} \ddot{q}_{j}-\dot{J}_{2, j}^{\operatorname{lin}} \dot{q}_{j}\right)+ \\
& \dot{u}^{T}\left(\dot{p}_{1, i}-\dot{p}_{2, j}\right) \geq \frac{1}{\Delta t}\left(-\xi \frac{d-\delta_{s}}{\delta_{i}-\delta_{s}}-\dot{d}\right) .
\end{aligned}
$$

See Fig. 4 for a schematic illustration of the behavior.

One limitation of this collision-avoidance approach is the possibility of the bodies to get stuck in local minima. The solution we retained for avoiding them is by letting the user specify, in the FSM described below, intermediate waypoints to guide the motion away from such local minimum if it occurs. One single way-point is in general sufficient and the user does not need to specify any explicit trajectory.

\subsection{Other Constraints of the Motion}

The next set of constraints is the unilateral contacts and friction cone constraints. A QP-compatible formulation of those is obtained by linearizing the friction cones into friction pyramids such that the forces $F_{0}$ and $F_{1}$ can be respectively written as $F_{0}=K_{0} \Lambda_{0}$ and $F_{1}=K_{1} \Lambda_{1}$, where $K_{0}$ and $K_{1}$ are the matrices of unit vectors generators of the pyramid edges, and $\Lambda_{0}$ and $\Lambda_{1}$ the coefficients along these generators. The unilateral and friction cones constraints become:

$$
\Lambda_{0} \geq 0 \text { and } \Lambda_{1} \geq 0 .
$$

We modeled two types of contacts with this contact framework: planar contacts and grasp contacts. Planar contact areas (e.g. foot sole) are modeled with 4 contact points and contact normals on a plane approximation of the contact area. Grasp contact are modeled with 4 contact points and normal vectors distributed along a cylindrical approximation of the hand palm and fingers contact area. We used 4-edge pyramid approximations of the friction cones, thus each contact of either type contributes with 16 variables in one of the two vectors $\Lambda_{0}$ and $\Lambda_{1}$. See Fig. 5 .

The last set of constraints are the position, velocity, and torque limits constraints

$$
\begin{aligned}
& q_{\min } \leq q \leq q_{\max }, \\
& \dot{q}_{\min } \leq \dot{q} \leq \dot{q}_{\max }, \\
& \tau_{\min } \leq \tau \leq \tau_{\max } .
\end{aligned}
$$

The joint limit constraint (26) is necessary for the geometrical consistency of the scene, while the torque limit constraint (28) can be enforced for its physical consistency if desired. The velocity limit constraint (27) is more inherited from robotics applications although not always relevant in a computer animation context. We include it in this description for completeness. QP-compatible formulations of inequalities (27) and (26) can be written respectively as

$$
\begin{aligned}
\frac{\dot{q}_{\min }-\dot{q}}{\Delta t} & \leq \ddot{q} \leq \frac{\dot{q}_{\max }-\dot{q}}{\Delta t}, \\
\frac{q_{\min }-q-\dot{q} \Delta t}{\frac{1}{2} \Delta t^{2}} & \leq \ddot{q} \leq \frac{q_{\max }-q-\dot{q} \Delta t}{\frac{1}{2} \Delta t^{2}} .
\end{aligned}
$$

However, the formulation (30) leads to strong decelerations when the joint comes close to its limit and to discontinuities in the torque output by the QP. To solve this we introduce a velocity damper similar to the one used for collision avoidance in the previous section. Let $d_{\min }=q-q_{\min }$ and $d_{\max }=q_{\max }-q$, we replace (30) with

$$
\frac{-\xi \frac{d_{\min }-\delta_{s}}{\delta_{i}-\delta_{s}}-\dot{q}}{\Delta t} \leq \ddot{q} \leq \frac{\xi \frac{d_{\max }-\delta_{s}}{\delta_{i}-\delta_{s}}-\dot{q}}{\Delta t},
$$

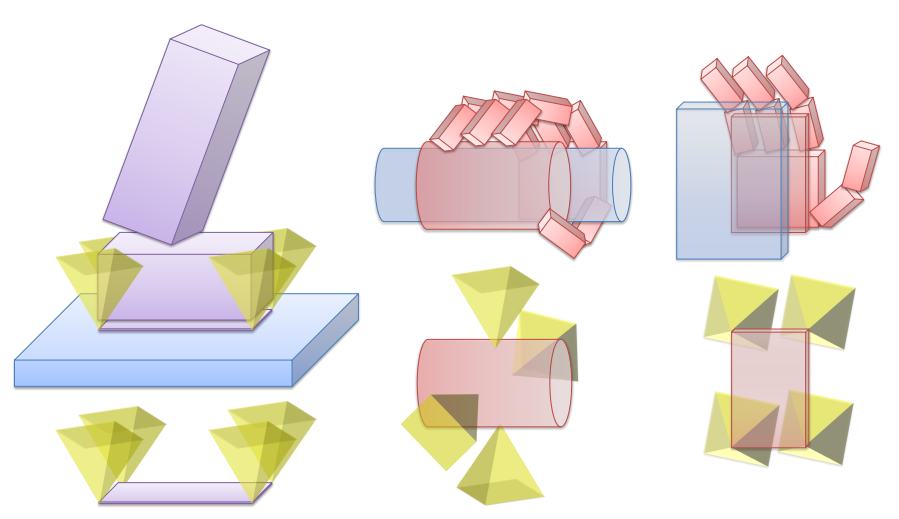

Fig. 5. Example of contact surfaces and contact point modeling. Feet contacts (left) are modeled as 4-point planar contacts. Hand grasp contact surfaces (middle) are modeled as cylindrical surfaces with 4 contact points around the surface (note that the pyramids are oriented to the outside for the hand-attached contact cylinder but are oriented to the inside of the cylinder for the object-attached cylindrical grasp surface, the latter are omitted in the figure for clarity). The hands can also be used for planar non-grasp surface (right) similarly to the feet or other planar contact areas such as the buttocks for sitting. 
which are added as constraints when $d_{\min } \leq \delta_{i}$ and $d_{\max } \leq$ $\delta_{i}$ respectively.

\subsection{Tasks/Objectives}

The tasks/objectives of the motion are expressed in terms of features of the system, a feature being any function $x(q)$ such as the position of the hand of the character, the trajectory of the foot of the character, the configuration of a door (opening angle), the position/orientation of a floating object, etc. A feature is associated with a Jacobian $J_{x}$ such that $\dot{x}=J_{x} \dot{q}$ and $\ddot{x}=J_{x} \ddot{q}+\dot{J}_{x} \dot{q}$. For a number $\mathcal{M}$ of simultaneous objectives $\left(x_{1}, \ldots, x_{\mathcal{M}}\right)$ in a given phase of the animation, the quadratic cost function to minimize in the $\mathrm{QP}$ is defined as

$$
c_{q, \dot{q}}(\ddot{q})=\sum_{m=1}^{\mathcal{M}} w_{m}\left\|\ddot{x}_{m}-\ddot{x}_{m}^{d}\right\|^{2},
$$

where $w_{m}$ are the relative weights of the objectives that are tuned by the user depending on which objective they would like to favor and depending on the observed behavior resulting from that choice (e.g. falling down would suggest increasing a COM objective weight); $\ddot{x}^{d}$ is a desired behavior that we borrow from the previous work [12] as

$$
\ddot{x}^{d}=-k\left(x-x^{\text {ref }}\right)-2 \sqrt{k}\left(\dot{x}-\dot{x}^{\text {ref }}\right)+\ddot{x}^{\text {ref }},
$$

where $x^{\text {ref }}$ is a reference trajectory that the user designs and would like to track, or a fixed value around which they would like to regulate the feature. $k$ is a defined stiffness gain for the task. In the example animations of this paper, it was sufficient to use only piecewise constant profiles of $x^{\text {ref }}$, i.e $\dot{x}^{\text {ref }}=0$ and $\ddot{x}^{\text {ref }}=0$ and thus

$$
\ddot{x}^{d}=-k\left(x-x^{\mathrm{ref}}\right)-2 \sqrt{k} \dot{x},
$$

both for regulating the feature $x$ around a constant value $x^{\text {reg }}\left(x^{\text {ref }}=x^{\text {reg }}\right)$ and for steering the feature $x$ to a distant target value $x^{\text {tgt }}\left(x^{\text {ref }}=x^{\text {tgt }}\right)$ (though we also implemented the target objective proposed in [13], we did not use it in our applications). In the coming Figures containing example objective descriptions (Figs. 7, 12, 13 later in the paper), we textually refer to both these kinds of tasks (regulating and

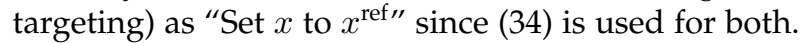

A typical phase of the animations we produced in the examples required the design of the following tasks:

- a reference rest pose for the whole configuration of the system. It can be rapidly sketched by the user giving a gross approximation of the expected postures during the motion, or obtained by means of inverse kinematics if the user wants a more refined pose, e.g. [42]. This task is typically lowweight task and used as a "background" task for regulating the values of the DOFs that are not used for the other tasks. In each of the demonstrated animation examples of this paper, only one rest pose was used both to initialize the system and for the whole motion.

- the reference/target COM of a character. We either regulate the COM around its stable static-pose value or its projection at the center of the support polygon, or we steer its projection away from a given contact area and into the reduced support polygon to remove that contact;
- the target $6 \mathrm{D}$ position and orientation of the swing foot of the characters in locomotion phases, including both the landing position of the foot and mid-step height,

- a target 3D position of the hand of a character for reaching tasks for example;

- a target 3D or $6 \mathrm{D}$ position of a rigid manipulated object, a target configuration/joint angle/position/orientation of an articulated part of the environment with which a character interacts;

- a reference/target COM of a group of characters/objects in contact with each other and moving together in physical interaction.

While all these tasks and features are classically used in existing QP controllers, the main novelty of our present work lies in the latter two tasks which are a specificity of our multi-character multi-object approach. Collaborative behav-

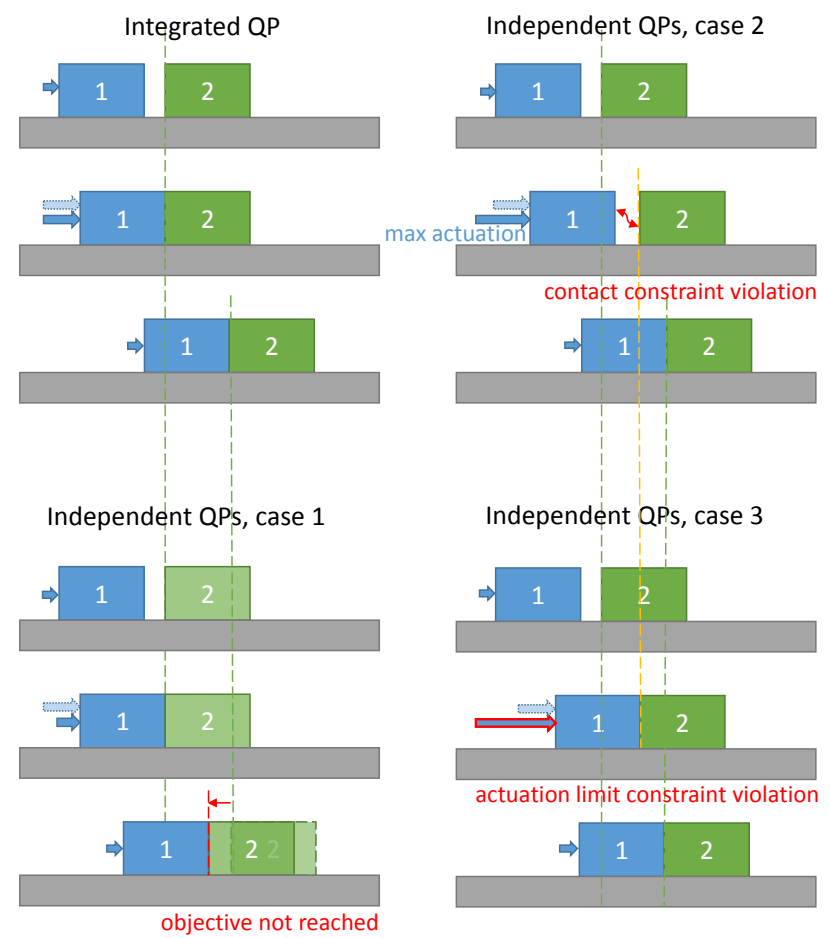

Fig. 6. The objective in this minimal theoretical case study is to use the multi-QP approach to manipulate the un-actuated object 2 with the actuated object 1 . The desired task by the user is on the final position of object 2. The integrated QP (top left) takes into account the coupling between the two objects and the inability of object 2 to move "by itself" to fulfill the desired position task. Various decoupled QP strategies with different orderings are compared in the three other cases. In case 1 (bottom left), a QP is first formulated for object 1, that is not aware of ("doesn't see") the mass added by object 2 . The QP for object 1 computes its motion to try to reach the same final position for itself as in the integrated QP case, but leading to a lesser actuation force (since the mass of object 2 is not accounted for) and to the final objective not reached in the physics simulation where the mass of object 2 slows down the motion. In the decoupled strategy of case 2 (top right), a kinematic QP is formulated for object 2 to follow the user-input desired task, and a dynamic QP is formulated for object 1 to try to follow the motion of object 2 in a physically-consitent manner. That QP for object 1 however reaches the actuation limit before reaching the contact, hence leading to the physically unrealistic aerial phase with the contact loss. Enforcing the kinematic contact constraint on the QP for object 1 to follow the output of the QP for object 2 leads to case 3 (bottom right) resulting in an acceleration and actuation force that violates the actuation limit. 


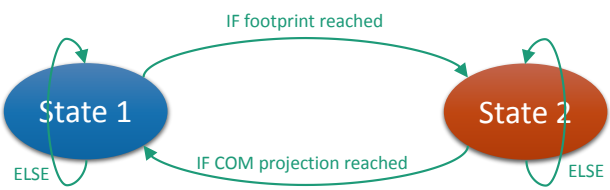

Set foot position to specified footstep

Set COM proj. at the center of the support polygon reduced from the footprint of the moving foot Set rest posture

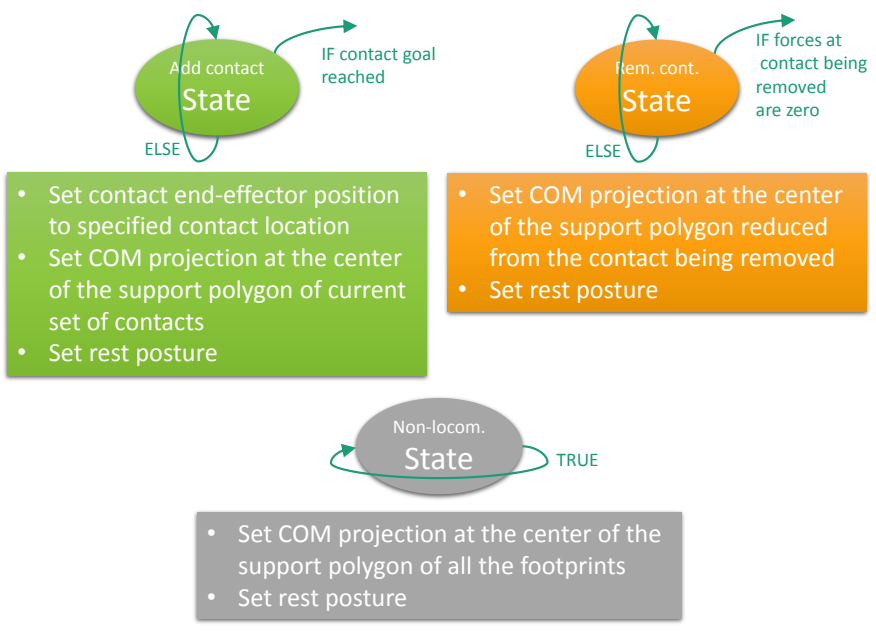

Set COM projection at the center of the support polygon reduced from the footprint of the next moving foot

Set rest posture

Non-cyclic locomotion-like behavior:

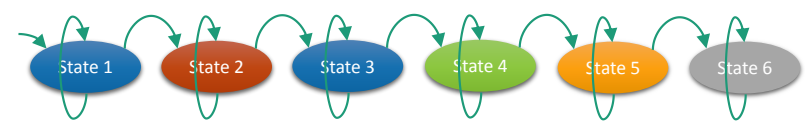

Fig. 7. FSM building-blocks with minimal sets of objectives. The two top states (blue and red) are the two states for cyclic locmotion alternating left foot and right foot steps. The two middle states (green and orange) are for general contact and grasp events (adding/removing). The bottom state (gray) is for non-locomotion phases (e.g. interactive reaching). Additional objectives can be added to these minimal sets of objectives depending on the particular scenario.

iors naturally emerge from all the characters present in the scene when controlling a feature that involves these characters. A single feature such as the position of a collaboratively manipulated heavy or bulky object will autonomously drive the behavior of all the characters that are in direct or indirect contact with this object or that are grasping a part of it. The same remark goes for the single COM of the system made of these characters and object. As previously introduced, these types of interactions and synergies among characters are part of the behavioral interactions that implicitly concur in the coupling of their motions.

\subsection{Final QP and FSM Controller}

The QP problem that is solved at every time-step of the simulation is formulated as follows

$$
\begin{aligned}
& \min _{\ddot{q}, \tau, \Lambda_{0}, \Lambda_{1}} c_{q, \dot{q}}(\ddot{q}) \\
& \text { subject to (12) (18) (20) (24) (25) (28) (29) (31). }
\end{aligned}
$$

At every control time-step $\Delta t$, the problem (35) is solved and the resulting $\ddot{q}$ is integrated to update the state $(q, \dot{q})$ of the system for the next time-step iteration.

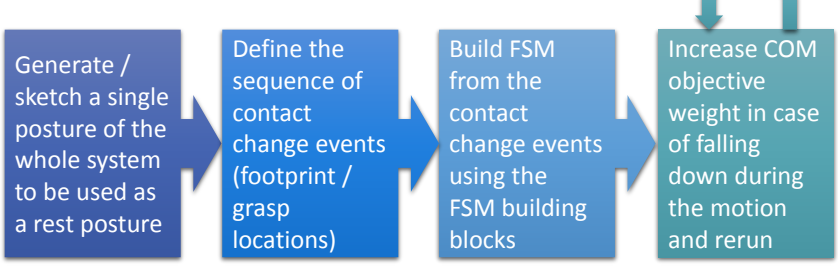

Fig. 8. Animation authoring workflow.

An alternative approach would have been to sequentially solve, within each iteration time-step, $n$ "small" QPs, one for each subsystem, rather than our integrated QP for the whole system. The contact forces and the positions of the contact points solved for QPs number 1 to $i$ fed as inputs to QP number $i+1$, and this iteratively for $i \in\{1, \ldots, n-1\}$. This approach would however prove sub-optimal and could lead to unfeasible problems whereas the one-QP approach would find a feasible solution. See Fig. 6 for a minimal theoretical example comparison between our integrated QP approach versus multi-QP strategies.

To create the final animation, the user needs to decompose the motion in phases within each of which the instance of the QP (35) remains the same, i.e phases with a fixed set of contacts and a fixed set of objectives, though the tracked target value of a given objective can vary in time within a given phase. A finite state machine (FSM) handles the transitions between the different phases of the motion (states of the FSM) when transition conditions are realized, e.g. foot landed, COM shifted, grasp established, grasp released, contact established, contact broken, etc.

Advanced FSM strategies as proposed in [13], [14] can also be used, though the use of [13] would require an additional effort in adapting its prioritized formulation to our weighted one (for example by assigning weights one order of magnitude higher for every priority level), especially for locomotion phases for which we just contented ourselves in the demonstrated examples with basic quasistatic, slow-gait, locomotion FSMs (alternating swinging feet and shifting COM projection on the new support polygon) for illustration purposes. See Figs. 7 and 8.

\section{Results}

We assessed our framework with original animation scenarios involving and incorporating multi-character interaction and cooperation, object manipulation, and interaction with the environment, see Fig. 9.

\section{King}

Two characters are lifting a third one sitting on a litter vehicle (king carrier). The whole system is made of 4 subsystems: The three characters (floating-base multi-body systems, $6+30 \mathrm{DOFs}$ each), and the litter vehicle (free-floating rigid object, 6 DOFs), adding up to a total of 114 DOFs for the system, of which 90 are actuated. The animation is decomposed into two phases: an autonomous locomotion and a user-interactive animations. The locomotion phase is decomposed into sub-phases representing the cyclic transitions between the two FSM states that are: 1) taking a step 

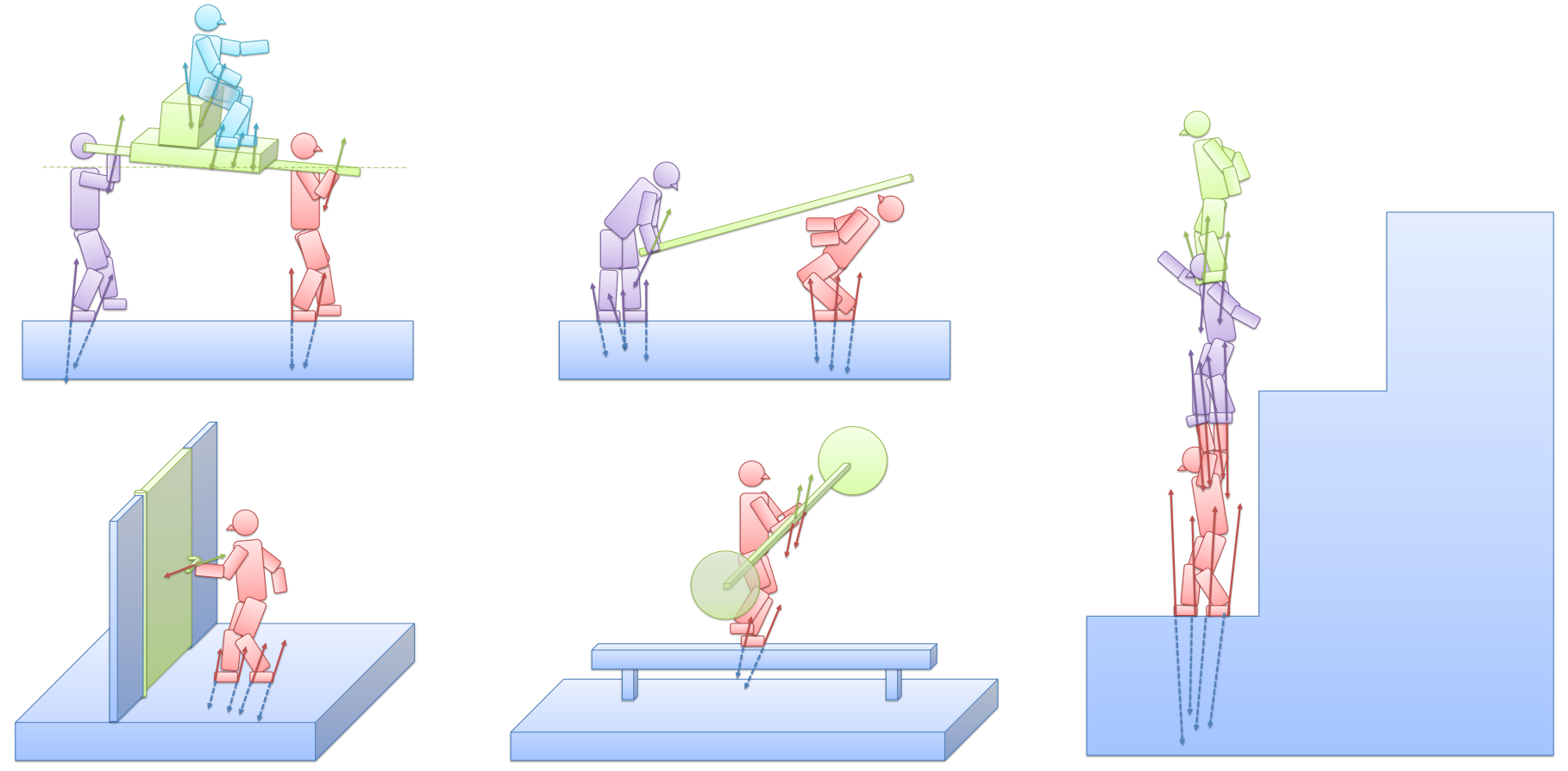

Fig. 9. Schematic representations of the demonstrated example scenarios. In each scenario each subsystem is represented in a different color and the contact forces applied on a given subsystem are represented in the same color as the subsystem (gravity forces are not represented for clarity).

by swinging the feet while keeping the projection of the COM of the whole system over the opposite feet support polygon, and 2) switching the COM of the whole system over the landed feet support polygon. In the interactive mode, the user controls the 3D $(x, y, z)$ position of a point in the scene that the carried character has to reach with her left hand (reaching task). The cooperative behavior of the two carriers in adjusting the position and orientation of the litter vehicle to ease the task for the third character emerges automatically, without any explicit specification.

\section{Funambulist}

A character walks along a narrow beam (width of the beam equals that of the character's foot), holding a barbell with randomly time-varying weights at each extremity. This is a locomotion-and-manipulation system made of two subsystems: the character $(6+30 \mathrm{DOFs})$ and the barbell (6 DOFs). The animation is decomposed into an autonomous locomotion phase and a user-interactive one. The locomotion phase is controlled by a cyclic two-state FSM as in Fig.7. The collisions between the legs during the swing phase are automatically avoided despite the constrained narrow line walking. When writing the FSM the user only has to worry about the foot landing position and mid-step height without providing collision-free trajectories. In the interactive mode, the arrows displayed on the scene are used to increase/decrease each of the two weights of the barbell. The character reacts in real-time making the adjustments in her posture to keep balance autonomously.

\section{Sword}

Two characters engage in an unfair battle with one of them equipped with a sword and the second one bare hands. The second character is however endowed with superior collision-avoidance capabilities that allow her to survive by dodging the swordsman's sword swipes while keeping balance. The system is composed of three subsystems: the two characters (30+6 DOFs each) and the sword (6 DOFs). The animation is decomposed into a scripted phase and a user-interactive phase. In the scripted phase the swordsman follows a sword trajectory pre-designed by the user, aiming first at the torso, then going back to the initial position, then aiming at the shoulder. In the user-interactive mode the arrows on the scene are used to control the movements of the sword, autonomously driving the movements of the swordsman without explicit control of his posture or his end-effector tasks. The dodging character's movements are fully autonomous and they all emerge from the two constraints: balance and collision avoidance. For this scene, a total of 33 collision-avoidance constraint pairs were added: 5 between the swordsman and his sword, 19 between his opponent and the sword, and 9 self-collision avoidance pairs for the dodging character.

\section{Acrobats}

Three characters team up in a three-story human tower building enterprise. For the purposes of this animation we dropped the torque limit constraint of the bottom character enabling her with intentional superhuman power. The system is made of the three characters $(30+6 \mathrm{DOF}$ each). The animation is decomposed into four meta-phases: 1) the top character climbs on the middle character, 2) the middle character carrying the top one climbs on the bottom character 3) the bottom character takes two steps while carrying the other two, and finally 4) a user-interactive phase. See Fig. 13 for the detailed FSM of the scenario. In the user interactive phase the user controls the $3 \mathrm{D}$ position of the COM of the top character, thus shaking the whole 
tower structure that manages to keep upright and to avoid collapsing by autonomously adjusting the postures and COMs of subsystems of characters.

\section{Door, Box, Valve, Lever}

The final animations demonstrate various kinds of interactions with the environment:

Door: The character opens and closes a door. The door is modeled as a 2-DOF fixed base multi-body system: 1 DOF for the hinges of the door and 1 DOF for the knob (both revolute joints, both un-actuated), the system has thus a total of $(30+6)+2=38$ DOFs of which 30 are actuated. The animation is decomposed into two phases: 1) rotating the knob and 2) rotating the door. The character avoids collision with the door and keeps balance during the rotation of the door automatically accounting for its moment of inertia. The task is only specified on the rotating angle of the door, and the motion of the character follows from that task.

Valve: The valve has also 2 DOFs, one revolute joint at the handle and one for the valve itself, the two joints having parallel axes this time. The task is to reach a desired angular velocity for the valve.

Box: The character operates a box by establishing planar contacts between her hands and two opposite surfaces of the boxes (not grasps). The system has $(30+6)+6=42$ DOFs (30 actuated) and 16 planar contact points (4 per foot/ground and 4 per hand/box). A random 6D trajectory of the box is specified and the character tracks it autonomously accounting for its mass and keeping balance.

Lever: The character operate a 1 DOF non-actuated lever equipped with passive spring-damper at its revolute joint and with an on-purpose voluminous part near its end. We have $(30+6+1)$ DOFs among which 30 are actuated and one is spring-loaded. The dynamical properties of the lever (moment of inertia, stiffness and damping of the spring) are included in its EOM and thus automatically accounted for in the animation. The task is only specified for the lever angle and the motion of the character follows while keeping balance and avoiding collision with the lever.

All these latter animations are scripted.

Tables 1 and 2 and Figures 10 and 11 present experimental figures for all the scenarios. The computation time figures were collected on a laptop Dell Alienware 14 with 7.7GiB memory, Intel Core i7-3720QM@2.60GHzx8, running under Ubuntu 12.04 64bits from a $\mathrm{C}++$ implementation of the framework. The EOMs, kinematics, and dynamics were computed using the implementations of the algorithms in [43]. Simulations were performed directly by integrating the resulting $\ddot{q}$ of the QP without using an external simulator. The QP solver used is LSSOL [44]. The generic humanoid character model (mass, inertia, link lengths, torque limits, joint angles and velocity limits) we used was an HRP-4 kinematics and dynamics model [45] with different geometric models, including the actual geometric model of HRP-4. Dynamics parameters for the other objects, door, valve, sword... were roughly estimated based on real-life objects and simple geometric model formulas.

\section{Discussion And Conclusion}

We presented a framework that greatly extends the scopes of applications of QP-based character controllers to animation
TABLE 1

Experimental figures for the example scenarios

\begin{tabular}{lccccc}
\hline & King & Funamb. & Sword & Acrobats & Door \\
\hline Nb. subsyst. & 4 & 2 & 3 & 3 & 2 \\
DOFs & 114 & 42 & 78 & 108 & 38 \\
Act. DOFs & 90 & 30 & 60 & 90 & 30 \\
Planar ctc. pts. & 28 & 8 & 16 & 24 & 8 \\
Grasp ctc. pts. & 16 & 8 & 8 & 0 & 4 \\
Nb. Col. pairs & 6 & 7 & 33 & 6 & 1 \\
QP size & $\mathbf{3 8 0}$ & $\mathbf{1 3 6}$ & $\mathbf{2 3 4}$ & $\mathbf{2 9 4}$ & $\mathbf{1 1 6}$ \\
Eq. constr. & 246 & 90 & 150 & 180 & 74 \\
Ineq. constr. & 298 & 99 & 317 & 282 & 93 \\
Min t (ms) & 4.45 & 0.89 & 0.76 & 5.97 & 0.77 \\
Max t (ms) & 84.51 & 5.10 & 14.97 & 34.88 & 2.33 \\
Med t (ms) & $\mathbf{2 2 . 7 2}$ & $\mathbf{1 . 2 9}$ & $\mathbf{6 . 1 2}$ & $\mathbf{8 . 1 0}$ & $\mathbf{0 . 8 9}$ \\
$\Delta t(\mathrm{~ms})$ & 33.33 & 5.00 & 10.00 & 10.00 & 5.00 \\
\hline
\end{tabular}

TABLE 2

Quantitative comparisons between this work (last column) and selected reference prior work: Abe et al. (2007) [12], Jain et al. (2009) [14] and de Lasa et al. (2010) [13]

\begin{tabular}{lcccc}
\hline & {$[12]$} & {$[14]$} & {$[13]$} & This \\
\hline Retained QP output & $\tau$ & $q$ & $\ddot{q}$ & $\ddot{\boldsymbol{q}}$ \\
Pb. size (char. DOFs) & 41 & 37 & 41 & $\mathbf{3 8}-\mathbf{1 1 4}$ \\
QP solver & MOSEK & SNOPT & MOS./QPC & LSSOL \\
Processor (Intel) & P4 & Core 2 & Xeon & Core i7 \\
QP control freq. (Hz) & 30 & 100 & 100 & $\mathbf{3 0 - 2 0 0}$ \\
Time-step (ms) & 33 & 10 & 10 & $\mathbf{5}-\mathbf{3 3}$ \\
Resol. time (ms) & $13-19$ & $100-500$ & $10-20$ & $\mathbf{0 . 9}-\mathbf{2 2}$ \\
Real-time & $100 \%$ & 2 to $10 \%$ & 50 to $100 \%$ & $\mathbf{1 0 0} \%$ \\
Multi-char. form. & no & unclear & no & yes \\
Reactive coll. av. & no & no & no & yes \\
\hline
\end{tabular}

scenarios beyond simple locomotion. We wrote all the EOMs of the characters, floating objects, articulated environment parts, as particular instances of the general multi-body system dynamics equation. We coupled them together through physical and behavioral interactions in the form of multicharacter-specific constraints or task objectives. We could thus adapt the multi-objective feature-based QP control approach to the control of the full system that is made up of all the moving and interacting elements/characters that appear in the scene. This systematic approach unlocks various horizons of possibilities offered by all imaginable combinations between characters and objects that it allows, creating animation scenarios that simultaneously and seamlessly integrate locomotion components, manipulation components, cooperative behaviors components, interactionamong-characters components, and interactions-with-theenvironment components.

The focus of this work was on the low-level controller. The latter was coupled with FSMs that decompose the scenarios into states with a fixed set of tasks and transition conditions between those states to change/add/remove tasks. Although simple FSMs were used mainly to serve as demonstrators of the performances of the low-level controller, this simplicity might have lead in some cases to oversimplifications that resulted in unrealistic behaviours. Such 


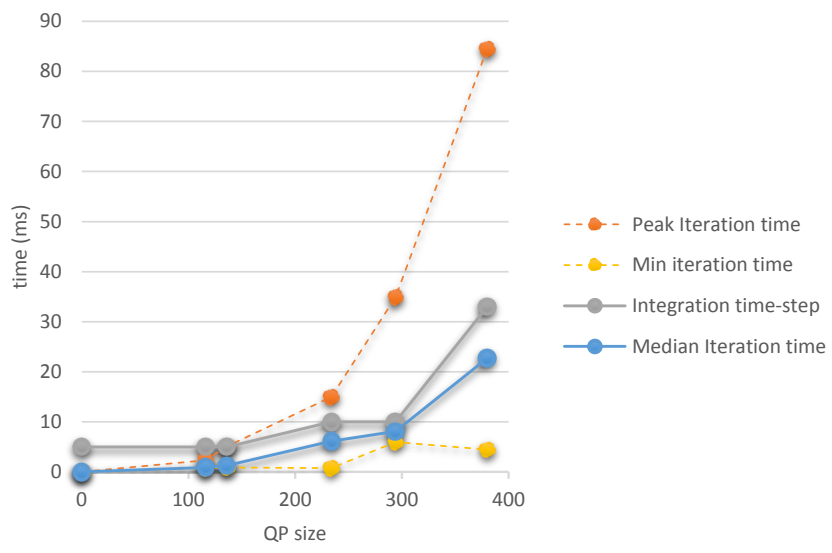

Fig. 10. A representation of the computation time requirements of our framework as a function of the complexity of the scenarios (represented here by the maximum QP size of the scenario, i.e. the total number of scalar variables in the QP of the scenario). The median iteration time (blue curve) is the most significant data as the peak iteration times (red dashed curve) are rarely reached and occur only at isolated points of time during the motion (mainly at the discrete contact change events). The integration time-step is adjusted to the median iteration time to keep real-time interactivity possible. From the profile of the blue curve we can expect that the framework would still have reasonable though non real-time computation times for significantly more complex scenarios if desired.

a one can be noted for example in the perfect coordination of the carriers' feet in the King scenario. In these cases a little more creativity effort would be required from the user in the FSM design.

The use of simple FSMs also caused two other limitations. First the balance criterion used throughout the demonstrated scenarios was a quasi-static one, controlling the ground projections of the COMs of the multi-characters system to their statically stable positions with setpoint tasks. Second, the absence of a look-ahead scheme in the controller prevents realizing more dynamic movements while staying balanced. These two limitations can be handled in the future by coupling the local QP controller presented here with a preview controller, in a model-predictive control (MPC) scheme (e.g. [19] or more recently in multi-contact behaviors [46]) on the COM of the full system and/or on the COMs of selected subsystems. A more challenging direction lies in increasing the level of autonomy of the framework by even sparring the user the design of the FSM itself and deriving this FSM from a planning phase, such as the idea in [35] with even higher-level objective specifications.

In this work, cooperating characters instantaneously communicate each other's "will" through the centralized QP rather than through vision, conversation, forces, etc. One additional improvement of the framework can be made by encoding the simulation of these realistic communication mean delays between the cooperating characters.

In additional future work, the computation times can be further substantially improved for larger problems by taking advantage of the sparsity of the QPs and hence using a solver that handles this property. Lastly, we plan to integrate motion-capture data in the framework by replacing the rest pose objective (reference posture) with the reference motion data tracking objective.
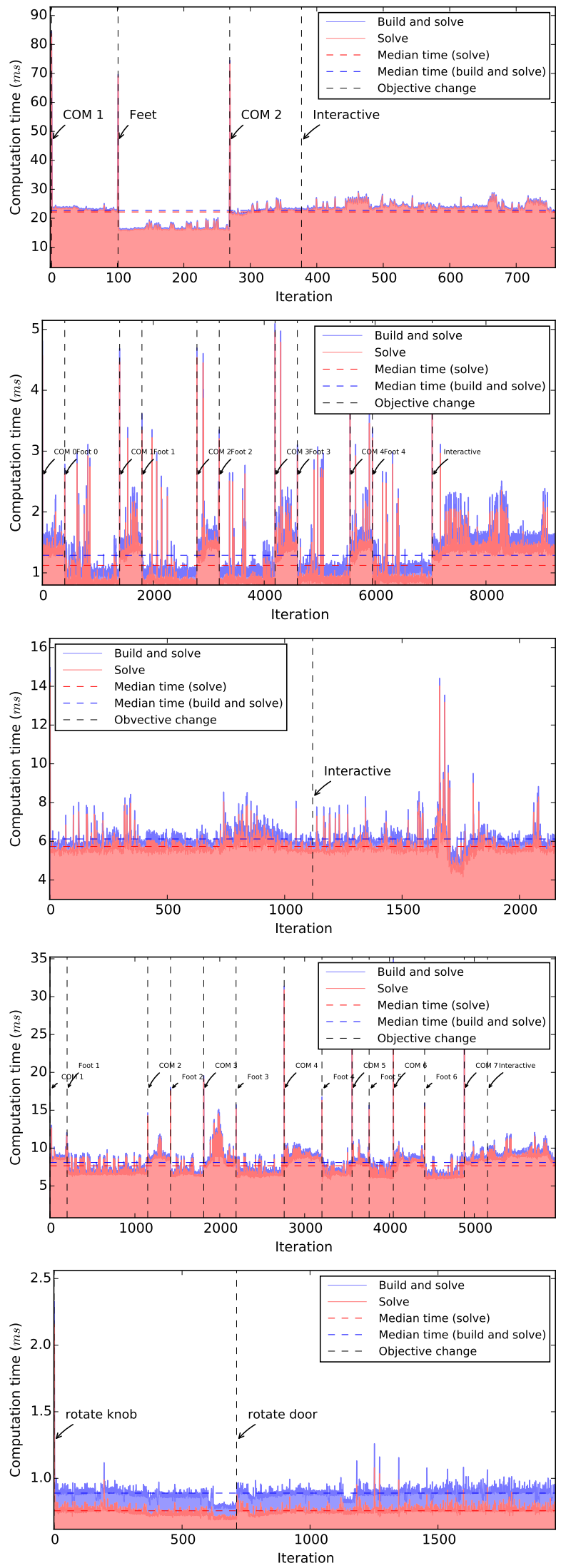

Fig. 11. Tracking of the computation times of each time-step of the example scenarios. From top to bottom: one step of the King scenario, the Funambulist scenario, the Sword scenario, the Acrobats scenarios, and the Door scenario. Vertical dashed lines represent FSM state transition events. 


\section{APPENDIX \\ FSM DETAILS}

See Figs 12 and 13.
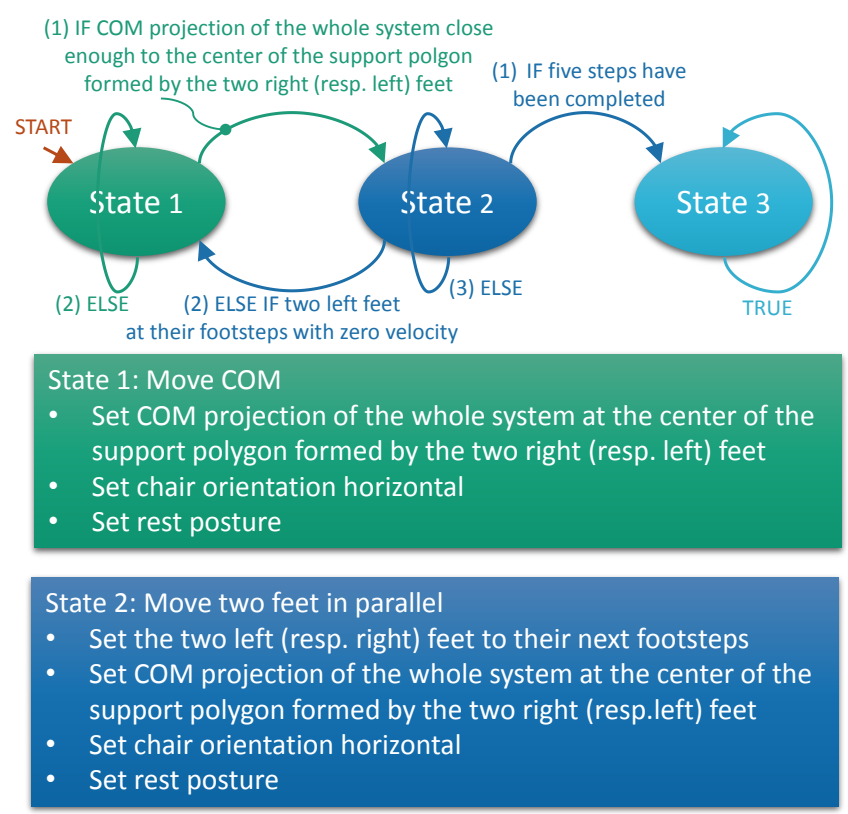

State 3: Interactive reaching

Set lifted character's left hand position at user cursor

Set COM projection of the whole system at the center of the support polygon formed by the four feet

Set rest posture

Fig. 12. Detailed FSM for the King scenario.

\section{REFERENCES}

[1] M. H. Raibert and J. K. Hodgins, "Animation of Dynamic Legged Locomotion," Computer Graphics (Proc. of SIGGRAPH 1991), vol. 25, no. 4, pp. 349-358, 1991.

[2] J. K. Hodgins, W. L. Wooten, D. C. Brogan, and J. F. O'Brien, "Animating Human Athletics," in Proc. of SIGGRAPH 1995, ser. Annual Conference Series, 1995, pp. 71-78.

[3] W. Wooten and J. Hodgins, "Simulating Leaping, Tumbling, Landing and Balancing Humans," in Proc. of IEEE Int. Conf. on Robotics and Automation (ICRA 2000), 2000, pp. 656-662.

[4] P. Faloutsos, M. van de Panne, and D. Terzopoulos, "Composable Controllers for Physics-based Character Animation," in Proc. of SIGGRAPH 2001, ser. Annual Conference Series, 2001, pp. 251260.

[5] K. Yin, K. Loken, and M. van de Panne, "SIMBICON: Simple Biped Locomotion Control," ACM Trans. on Graphics (Proc. of SIGGRAPH 2007), vol. 26, no. 3, p. 105, 2007.

[6] Y.-Y. Tsai, W.-C. Lin, K. B. Cheng, J. Lee, and T.-Y. Lee, "RealTime Physics-Based 3D Biped Character Animation Using an Inverted Pendulum Model," IEEE Trans. Visualization and Computer Graphics, vol. 16, no. 2, pp. 325-337, 2010.

[7] A. Liegeois, "Automatic Supervisory Control of the Configuration and Behavior of Multibody Mechanisms," IEEE Trans. on Systems, Man, and Cybernetics, vol. 7, no. 12, pp. 868-871, 1977.

[8] O. Khatib, "A Unified Approach for Motion and Force control of Robot Manipulators: The Operational Space Formulation," IEEE Journal of Robotics and Automation, vol. 3, no. 1, pp. 43-53, 1987.

[9] L. Sentis and O. Khatib, "Synthesis of Whole-Body behaviors Through Hierarchical Control of Behavioral Primitives," International Journal of Humanoid Robotics, vol. 2, no. 4, pp. 505-518, 2005.

[10] L. Righetti, J. Buchli, M. Mistry, and S. Schaal, "Inverse dynamics control of floating-base robots with external constraints: A unified view."
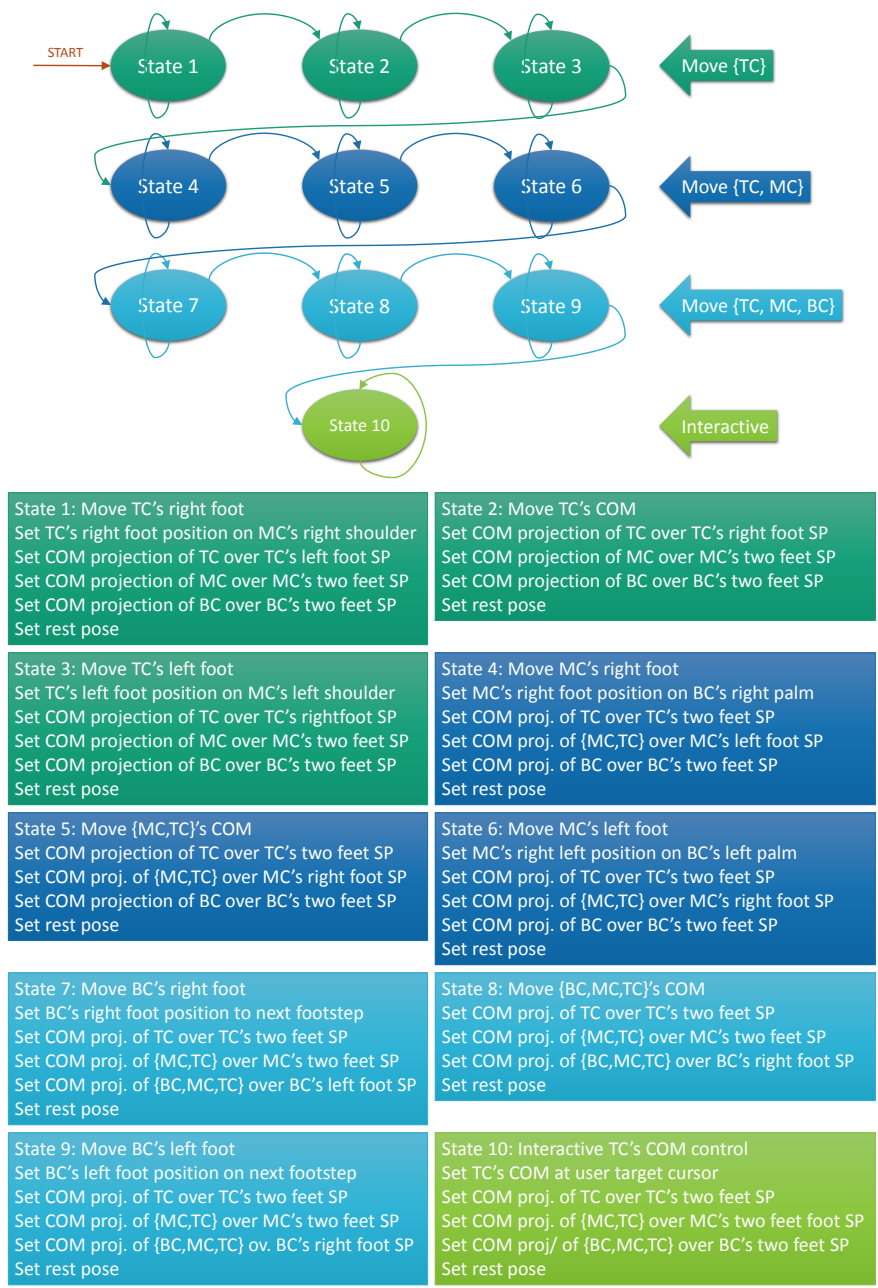

Fig. 13. Detailed FSM for the Acrobats scenario. For clarity we only detail the state objectives and omit the transition conditions. Abbreviations used int he descriptions of objectives: SP Support Polygon, TC: Top Character, MC: Middle Character, BC: Bottom Character.

[11] Y. Abe and J. Popović, "Interactive Animation of Dynamic Manipulation," in Proc. of the ACM SIGGRAPH/Eurographics symposium on Computer animation. Eurographics Association, 2006, pp. 195204.

[12] Y. Abe, M. da Silva, and J. Popović, "Multiobjective Control with Frictional Contacts," in Proc. of the ACM SIGGRAPH/Eurographics symposium on Computer animation. Eurographics Association, 2007, pp. 249-258.

[13] M. de Lasa, I. Mordatch, and A. Hertzmann, "Feature-Based Locomotion Controllers," ACM Trans. on Graphics (Proc. of SIGGRAPH 2010), vol. 29, no. 3, p. 131, 2010.

[14] S. Jain, Y. Ye, and C. K. Liu, "Optimization-Based Interactive Motion Synthesis," ACM Trans. on Graphics, vol. 28, no. 1, p. 10, 2009.

[15] M. da Silva, Y. Abe, and J. Popović, "Interactive Simulation of Stylized Human Locomotion," ACM Trans. on Graphics (Proc. of SIGGRAPH 2008), vol. 27, no. 3, p. 82, 2008.

[16] — " "Simulation of Human Motion Data using ShortHorizon ModelPredictive Control," Computer Graphics Forum, vol. 27, no. 2, pp. 371-380, 2008.

[17] A. Macchietto, V. Zordan, and C. R. Shelton, "Momentum Control for Balance," ACM Trans. on Graphics (Proc. of SIGGRPAH 2009), vol. 28, no. 3, p. 80, 2009.

[18] M. Al Borno, E. Fiume, A. Hertzmann, and M. de Lasa, "Feedback Control for Rotational Movements in Feature Space," Computer Graphics Forum, vol. 33, no. 2, pp. 225-233, 2014.

[19] I. Mordatch, M. De Lasa, and A. Hertzmann, "Robust PhysicsBased Locomotion Using Low-Dimensional Planning," ACM 
Trans. on Graphics (Proc. of SIGGRAPH 2011), vol. 29, no. 4, p. 71, 2010.

[20] C. K. Liu and Z. Popović, "Synthesis of Complex Dynamic Character Motion from Simple Animations," ACM Trans. on Graphics, vol. 21, no. 3, pp. 408-416, 2003.

[21] A. C. Fang and N. S. Pollard, "Efficient Synthesis of Physically Valid Human Motion," ACM Trans. on Graphics (Proc. of SIGGRAPH 2003), vol. 22, no. 3, pp. 417-426, 2003.

[22] M. Al Borno, M. de Lasa, and A. Hertzmann, "Trajectory Optimization for Full-Body Movements with Complex Contacts," IEEE Trans. on Visualization and Computer Graphics, vol. 19, no. 8, pp. 1405-1414, 2013.

[23] Y. Koga, K. Kondo, J. J. Kuffner, and J.-C. Latombe, "Planning Motions with Intentions," in Proc. of SIGGRAPH 1994, ser. Annual Conference Series, 1994, pp. 395-408.

[24] K. Yamane, J. Kuffner, and J. K. Hodgins, "Synthesizing Animations of Human Manipulation Tasks," ACM Trans. on Graphics (Proc. of SIGGRAPH 2004), vol. 23, no. 3, pp. 532-539, 2004.

[25] S. Jain and C. K. Liu, "Interactive Synthesis of Human-Object Interaction," in Proc. of the ACM SIGGRAPH/Eurographics symposium on Computer animation. Eurographics Association, 2009, pp. 47-53.

[26] Y. Bai, K. Siu, and C. K. Liu, "Synthesis of Concurrent Object Manipulation Tasks," ACM Trans. on Graphics (Proc. of SIGGRAPH Asia 2012), vol. 31, no. 6, p. 156, 2012.

[27] C. Esteves, G. Arechavaleta, J. Pettré, and J.-P. Laumond, "Animation Planning for Virtual Characters Cooperation," ACM Trans. on Graphics, vol. 25, no. 2, pp. 319-339, 2006.

[28] K. Wampler, E. Andersen, E. Herbst, Y. Lee, and Z. Popović, "Character Animation in Two-Player Adversarial Games," ACM Trans. on Graphics, vol. 29, no. 3, p. 26, 2010.

[29] H. P. H. Shum, T. Komura, and S. Yamazaki, "Simulating Multiple Character Interactions with Collaborative and Adversarial Goals," IEEE Trans. Visualization and Computer Graphics, vol. 18, no. 5, pp. 741-752, 2012.

[30] C. K. Liu, A. Hertzmann, and Z. Popović, "Composition of Complex Optimal Multi-Character Motions," in Proc. of the ACM SIGGRAPH/Eurographics symposium on Computer animation. Eurographics Association, 2006, pp. 215-222.

[31] S. Coros, A. Karpathy, B. Jones, L. Reveret, and M. Van De Panne, "Locomotion Skills for Simulated Quadrupeds," ACM Trans. on Graphics (Proc. of SIGGRAPH 2011), vol. 30, no. 4, p. 59, 2011.

[32] C. K. Liu, "Dextrous Manipulation from a Grasping Pose," $A C M$ Trans. on Graphics, vol. 28, no. 3, p. 59, 2009.

[33] I. Mordatch, E. Todorov, and Z. Popović, "Discovery of Complex Behaviors Through Contact-Invariant Optimization," ACM Trans. on Graphics (Proc. of SIGGRAPH 2012), vol. 31, no. 4, p. 43, 2012.

[34] _ "Contact-Invariant Optimization for Hand Manipulation," in Proc. of the ACM SIGGRAPH/Eurographics symposium on Computer animation. Eurographics Association, 2012, pp. 137-144.

[35] K. Bouyarmane and A. Kheddar, "Multi-Contact Stances Planning for Multiple Agents," in Proc. of the IEEE Int. Conf. on Robotics and Automation (ICRA 2011), 2011, pp. 5546-5353.

[36] — "Static Multi-Contact Inverse Problem for Multiple Humanoid Robots and Manipulated Objects," in Proc. of the IEEE-RAS Int. Conf. on Humanoid Robots (Humanoids 2010), 2010, pp. 8-13.

[37] A. Clegg, J. Tan, G. Turk, and C. K. Liu, "Animating human dressing," ACM Trans. on Graphics (Proc. of SIGGRAPH 2015), vol. 34, no. 4, pp. 116:1-116:9, Jul. 2015.

[38] M. Peinado, D. Meziat, D. Maupu, D. Raunhardt, D. Thalmann, and R. Boulic, "Accurate on-line avatar control with collision anticipation," in Proceedings of the 2007 ACM Symposium on Virtual Reality Software and Technology, ser. VRST '07. New York, NY, USA: ACM, 2007, pp. 89-97.

[39] A. Escande, S. Miossec, M. Benallegue, and A. Kheddar, "A Strictly Convex Hull for Computing Proximity Distances with Continuous Gradient," IEEE Trans. on Robotics, vol. 30, no. 3, pp. 666-678, 2014.

[40] B. Faverjon and P. Tournassoud, "A Local Based Approach for Path Planning of Manipulators with a High Number of Degrees of Freedom," in IEEE Int. Conf. on Robotics and Automation, March 1987, pp. 1152-1159.

[41] F. Kanehiro, F. Lamiraux, O. Kanoun, E. Yoshida, and J.-P. Laumond, "A Local Collision Avoidance Method for Non-Strictly Convex Polyhedra," in Robotics: Science and Systems IV, Zurich, Switzerland, June 2008.

[42] P. Baerlocher and R. Boulic, "An Inverse Kinematics Architecture Enforcing an Arbitrary Number of Strict Priority Levels," The Visual Computer, vol. 20, no. 6, pp. 402-417, 2004.
[43] R. Featherstone, Rigid Body Dynamics Algorithms. Secaucus, NJ, USA: Springer-Verlag New York, Inc., 2007.

[44] P. E. Gill, S. J. Hammarling, W. Murray, M. A. Saunders, and M. H. Wright, "User's Guide For LSSOL (Version1.0): A Fortran Package for Constrained Linear Least-Squares and Convex Quadratic Programming," Stanford University, Department of Operations Research, Tech. Rep., 1986.

[45] K. Kaneko, F. Kanehiro, M. Morisawa, K. Akachi, G. Miyamori, A. Hayashi, and N. Kanehira, "Humanoid Robot HRP-4 Humanoid Robotics Platform with Lightweight and Slim Body", in Proc. of IEEE/RSJ Int. Conf. on Intelligent Robots and Systems (IROS 2011), 2011, pp. 4400-4407.

[46] H. Audren, J. Vaillant, A. Kheddar, A. Escande, K. Kaneko, and E. Yoshida, "Model Preview Control in Multi-Contact Motion Application to a Humanoid Robot," in Proc. of the IEEE/RSJ Int. Conf. on Intelligent Robots and Systems (IROS 2014), 2014, pp. 40304035.

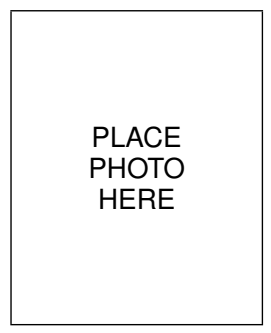

Joris Vaillant received the Master degree in interactive systems at Paul Sabatier University, Toulouse, France, in 2010 and the PhD degree in robotics from the University of Montpellier in 2015. His research interest is contact planning and whole body motion for humanoids and virtual avatars.

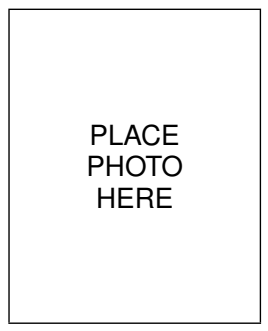

Karim Bouyarmane received the Ingenieur diploma from Ecole Polytechnique in Palaiseau in 2007 and from Ecole Nationale Superieure des Mines de Paris in 2008, and the PhD degree from the University of Montpellier in 2011 He completed the PhD program in the Joint Robotics Laboratory (JRL) at the National Institute of Advanced Industrial Science and Technology (AIST) in Tsukuba, Japan. He then held a Japan Society for the Promotion of Science (JSPS) post-doctoral fellowship at the Advanced Telecommunications Research Institute International (ATR) in Kyoto, in Mitsuo Kawato's Computational Neuroscience Laboratories working on brain-robot interfaces. He is currently a CNRS research associate.

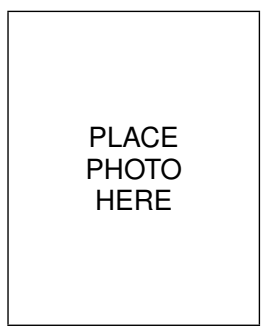

Abderrahmane Kheddar received the BSCS degree from the Institut National dlnformatique (ESI), Algiers, the $\mathrm{MSc}$ and $\mathrm{PhD}$ degrees in robotics, both from the University of Pierre and Marie Curie, Paris 6 . He is presently Directeur de Recherche at CNRS. He is the Director of the CNRS-AIST Joint Robotic Laboratory (JRL), UMI3218/RL, Tsukuba, Japan; and the leader of the Interactive Digital Humans (IDH) team at CNRS-UM LIRMM at Montpellier, France. His research interests include humanoid robotics, haptics, and thought-based control. He is a founding member of the IEEE/RAS chapter on haptics (now acting as a senior advisor), the cochair and co-founding member of the IEEE/RAS Technical committee on model-based optimization. He is presently Editor of the IEEE Transactions on Robotics, and the Journal of Intelligent and Robotic Systems. $\mathrm{He}$ is a founding member of the IEEE Transactions on Haptics and served in its editorial board during three years (2007-2010), he also served as associate editor in the MIT Press PRESENCE journal. $\mathrm{He}$ coordinated or acted as a PI for several EU projects. He is titular (full) member of the National Academy of Technologies of France (NATF) and a Senior Member of the IEEE Society. 